\title{
Decay of Metastable States: Sharp Transition from Quantum to Classical Behavior
}

\author{
D. A. Gorokhov and G. Blatter \\ Theoretische Physik, ETH-Hönggerberg, CH-8093 Zürich, Switzerland \\ e-mail: gorokhov@itp.phys.ethz.ch
}

\begin{abstract}
The decay rate of metastable states is determined at high temperatures by thermal activation, whereas at temperatures close to zero quantum tunneling is relevant. At some temperature $T_{c}$ the transition from classical to quantum-dominated decay occurs. The transition can be first-order like, with a discontinuous first derivative of the Euclidean action, or smooth with only a second derivative developing a jump. In the former case the crossover temperature $T_{c}$ cannot be calculated perturbatively and must be found as the intersection point of the Euclidean actions calculated at low and high temperatures. In this paper we present a sufficient criterion for a first-order transition in tunneling problems and apply it to the problem of the tunneling of strings. It is shown that the problem of the depinning of a massive string from a linear defect in the presence of an arbitrarily strong dissipation exhibits a first-order transition.
\end{abstract}

PACS numbers: 64.60.My, 74.50.+r, 74.60.Ge

\section{INTRODUCTION}

Investigations of the decay rate of metastable states have a long history, going back to Kramers 1 who calculated the lifetime of a classical trapped particle separated from the true equilibrium state by a high potential barrier Since then different decay phenomena have been investigated. The motion of dislocations acress the Peierls barrier 23 the decay of the current in a Josephson loop, $\$$ and the creep of vortices in superconductors 5 are typical and well-known examples of metastability. At sufficiently high temperatures a metastable system decays due to thermal activation and the decay rate $\Gamma$ obeys the Arrhenius law $\Gamma \sim \exp (-U / T)$, whereas at temperatures close to absolute zero quantum tunneling is relevant and $\Gamma \sim \exp (-S / \hbar)$, with $S$ the action at zero temperature. Below we shall consider systems for which the semiclassical description is applicable. In this case $U / T$ and $S / \hbar$ are large; otherwise, the system would not be truly metastable.

Let $\mathbf{u}(t, \mathbf{r})$ denote the coordinates of the system under consideration depending in general on imaginary time $t$ and spatial variables $\mathbf{r}$. Within the semiclassical approximation the decay rate at a temperature $T$ is determined by the contribution of the trajectories close to the one extremizing the Euclidean action and satisfying the periodicity condition $\mathbf{u}(0, \mathbf{r})=\mathbf{u}(\hbar / T, \mathbf{r})$. In the high-temperature regime the function extremizing the action is time independent and, consequently, the activation barrier in the Arrhenius law $U$ does not depend on the dynamic properties of the system, meanwhile the bounce solution at low temperatures is time-dependent and the dynamics enters. At some temperature $T_{c}$ the transition from the time-independent to the time-dependent solution occurs. In some cases the bounce solution just below the crossover point can be written in the form

$$
\mathbf{u}(t, \mathbf{r})=\mathbf{u}_{t h}(\mathbf{r})+\delta \mathbf{u}(\mathbf{r}) \cos \left(\frac{2 \pi T_{c} t}{\hbar}\right)
$$

where the function $\delta \mathbf{u}(\mathbf{r})$ is considered to be small. Such an assumption leads to an action $S_{\text {Eucl }}(T)$ with a continuous first derivative in the crossover point (see Fig. 1).

The second derivative of $S$ is discontinuous at the point $T_{c}$. The second derivative has a jump at $T=T_{c}$. Following Ref. 3 we shall call this situation a "second-order transition in the crossover point." In this case the bounce solution with the minimal Euclidean action at low temperatures can be obtained by a continuous deformation of the thermal solution. However, in general such a deformation is not possible: The trajectory corresponding to the minimal action may jump at a certain temperature. In this case we deal with a first-order transition: it can be shown that the first derivative of $S_{\mathrm{Eucl}}(T)$ has a jump at the transition point (see Fig. 2) and the expansion (11) is not valid.

Strictly speaking, even if we deal with a first-order transition in a tunneling problem, there is a narrow crossover region from one solution to another because contributions originate from several saddle points and we need to take into account all of them. However, the better the semiclassical approximation is applicable, the narrower this region becomes. 
The problem considered here is related to the mean-field theory of phase transitions. The Euclidean action can be identified with the free energy in the Landau theory with an order parameter $\delta(\mathbf{r}, T) \operatorname{defined}_{\text {as }} \delta(\mathbf{r}, T)=\max _{t} \mid \mathbf{u}(t, \mathbf{r})-$ $\mathbf{u}_{t h}(\mathbf{r}) \mid$, for example. At high temperatures $\mathbf{u}(t, \mathbf{r})=\mathbf{u}_{t h}(\mathbf{r})$ and $\delta(\mathbf{r}, T) \equiv 0$, whereas at $T<T_{c}, \delta(\mathbf{r}, T) \neq 0$. The order parameter changes continuously at the point $T=T_{c}$ in the case of a second-order transition and discontinuously if a first-order transition takes place.

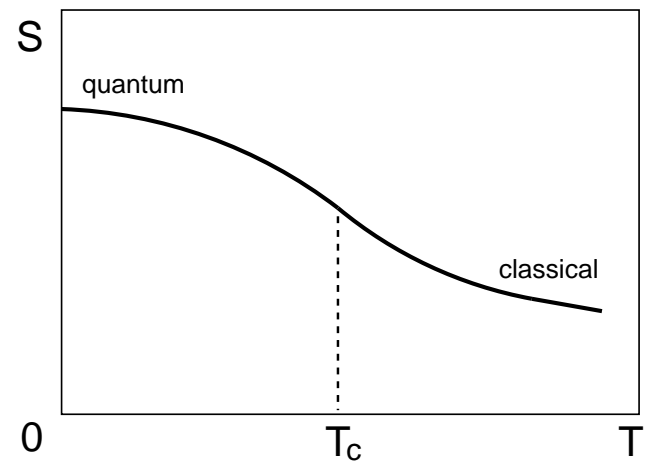

Fig.1 Euclidean action $S$ as a function of temperature $T$ for the case of a second-order transition.

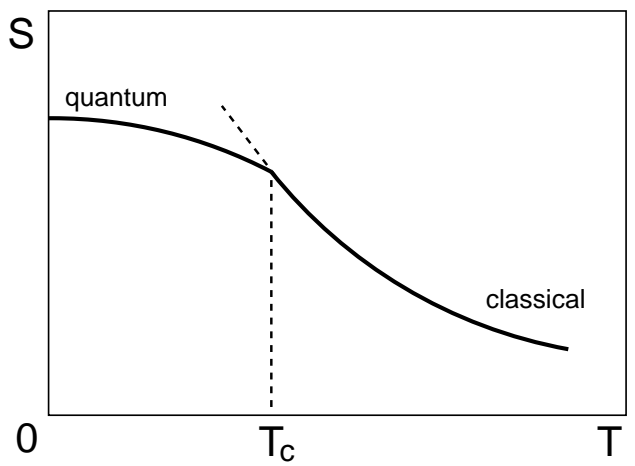

Fig.2 Euclidean action $S$ as a function of temperature $T$ for the case of a first-order transition. The first derivative of $S$ is discontinuous at the point $T_{c}$.

In this paper we present a simple criterion for the appearance of a first-order transition from quantum to classical behavior in tunneling problems and apply it to the problem of the tunneling of strings.

\section{OSCILLATIONS CLOSE TO THE THERMAL SADDLE-POINT SOLUTION}

In this section we study the behavior of the imaginary time oscillation period of the solution of the equation of motion close to the thermal saddle-point solution. In section II A we describe the general theory for one-dimensional (1D) Hamiltonian systems. We briefly summarize the results of Lifshitz and Kagand and Chudnovsky for 1D Hamiltonian systems and provide an expression for the jump of the derivative of the Euclidean action (see Eq. (13)) for the case of a first-order transition. In section II B we use a perturbative approach for the calculation of the imaginary time oscillation period in the vicinity of the thermal saddle-point solution, and define a criterion for a first-order transition.

\section{A. General theory for 1D Hamiltonian systems}

As shown by Chudnovsky 8 , if the imaginary time oscillation period of a massive particle is not a monotonous function of energy, a first-order transition from quantum to classical behavior takes place. This statement remains 
true for any one-dimensional Hamiltonian system: Consider a 1D system subject to potential which allows tunneling in one direction (Fig. 3).

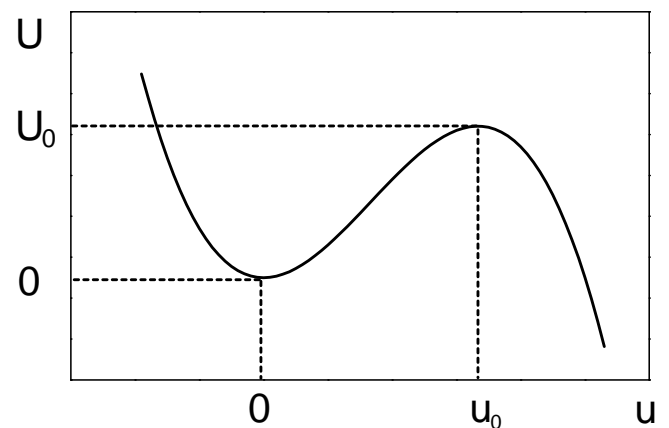

Fig.3 1D potential well with a metastable state. A particle trapped at $u=0$ can tunnel out to the right in order to reach the true ground state.

We choose the metastable minimum of the potential as our zero energy. Let us define the height of the potential barrier as $U_{0}$. Using the semiclassical approximation we can write the imaginary parts $\hbar \Gamma_{n}$ of the energy $E_{n}$ of the eigenstates in the form 9

$$
\hbar \Gamma_{n}=\frac{\hbar \omega\left(E_{n}\right)}{4 \pi} \exp \left(-\frac{2}{\hbar} \int_{a_{n}}^{b_{n}}|p| d x\right) \equiv \frac{\hbar \omega\left(E_{n}\right)}{4 \pi} \exp \left(-\frac{S_{n}}{\hbar}\right)
$$

where $p$ is the generalized momentum, $E_{n}<U_{0}$ are the energy levels in the equivalent potential without tunneling, and $\omega\left(E_{n}\right)$ are the corresponding classical oscillation frequencies. At finite temperature $T$ the decay rate can be easily found by averaging over the Boltzmann distribution

$$
\Gamma=\frac{2}{Z} \sum_{n} \frac{\omega\left(E_{n}\right)}{4 \pi} \exp \left(-\frac{E_{n}}{T}-\frac{S_{n}}{\hbar}\right)
$$

where $Z=\sum_{n} \exp \left(-\frac{E_{n}}{T}\right)$ is the partition function of the particle in the well. With Eq. (3) we reproduce the well-known expression $\Gamma=(2 / \hbar) \operatorname{Im} F$ for the decay rate at low $T$ (see Ref 10 ).

Equation (3) is applicable only at sufficiently low temperatures. At high temperatures the excitations with energies larger than the barrier height are relevant in the determination of the preexponential factor. The decay rate then is given by the equation $1,11.12$

$$
\Gamma=\frac{\omega\left(E_{0}\right)}{2 \pi} \exp \left(-\frac{U_{0}}{T}\right)
$$

where $\omega\left(E_{0}\right)$ is the oscillation frequency of the system near the metastable minimum. It is necessary to emphasize that the above expressions for the decay rate are applicable in the case of intermediate friction $\eta$. E.g., for a particle of mass $m$ the criterion $\omega T / U_{0} / m \ll \omega$, where $\omega^{2}=-U^{\prime \prime}\left(u_{0}\right) / m$ with $u_{0}$ the position of the maximum of the metastable potential (see Refs.1.11.12), guarantees, that on the one hand the system in the well is properly equilibrated, while on the other hand the dynamics is not affected by the damping.

The sum in Eq. (3) can be well approximated by the term where the function $f_{T}(E)=-E / T-S(E) / \hbar$ takes its maximal value and the problem reduces to the calculation of the maximum of $f_{T}(E)$ within the interval $\left[0, U_{0}\right]$. The extremal condition for $f_{T}(E)$ takes the form

$$
\frac{d f_{T}}{d E}=-\frac{1}{T}-\frac{1}{\hbar} \frac{d S}{d E}=0 .
$$

It is well known from standard courses of classical mechanics 13 that for a periodic problem the derivative of the action with respect to the energy is equal to the oscillation time $\tau$ at this energy. In our case the energy axis has the opposite direction (the action $S(E)$ corresponds to the motion in the inverse potential with energy $E$ ). Hence,

$$
\frac{d S}{d E}=-\frac{\hbar}{T}=-\tau(E)
$$


i.e., the decay is dominated by the Euclidean action with the trajectory periodic in the time interval $\hbar / T$.

If the function $\tau(E)$ is a monotonously decreasing function of energy, Eq. (6) has one solution for $T<\hbar / \tau_{0}$ $\left(\tau_{0}=\tau\left(U_{0}\right)\right)$ and no solutions for $T>\hbar / \tau_{0}$. At temperature $T_{c}=\hbar / \tau_{0}$ a second-order transition takes place.

Next, let us suppose that $\tau(E)$ goes through a minimum in the interval $\left(0, U_{0}\right)$ (see Fig. 4). In this case it can be shown 1 that there is an energy $E_{1} \in\left(0, E_{\min }\right)$, and associated with it a temperature $T_{c}=\hbar / \tau\left(E_{1}\right)$, where a first-order transition takes place.

Let us show that if $E_{1} \neq U_{0}$, the Euclidean action $S_{\text {Eucl }}(T)$ determining the decay rate $\Gamma$ indeed has a discontinuous derivative at the point $T=T_{c}$ (see Eq. (何). At $T>T_{c}$

$$
\frac{S_{\text {Eucl }}(T)}{\hbar}=\frac{S_{\text {thermal }}(T)}{\hbar}=\frac{U_{0}}{T}
$$

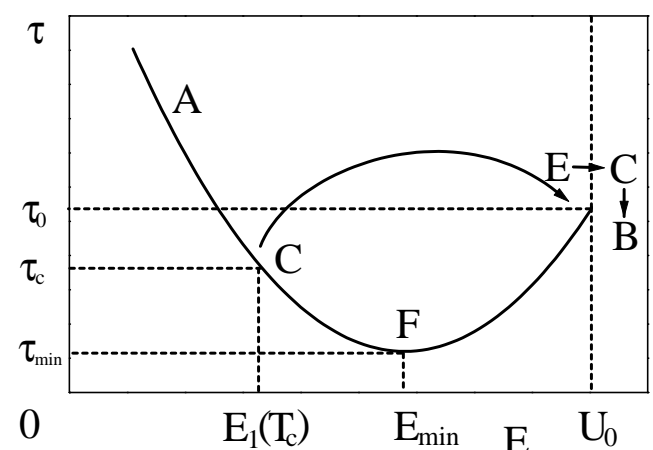

Fig.4 Non-monotonous dependence of the oscillation time $\tau$ in the inverse potential on the energy $E$. The arrow indicates the jump of the semiclassical trajectory from the thermal assisted quantum solution to the classical one.

The action just below $T_{c}$ can be written as (see Eq. (3))

$$
\frac{S_{\text {Eucl }}(T)}{\hbar}=\frac{S_{\text {quantum }}(T)}{\hbar}=\frac{E_{1}(T)}{T}+\frac{S\left(E_{1}\right)}{\hbar}
$$

(note that $E_{1}$ is a function of temperature).

The variations of $S_{\text {thermal }}$ and $S_{\text {quantum }}$ with respect to the $T$ near the point $T_{c}$ are

$$
\begin{gathered}
\frac{\delta S_{\text {thermal }}}{\hbar}=-\frac{U_{0}}{T_{c}{ }^{2}} \delta T \\
\frac{\delta S_{\text {quantum }}}{\hbar}=-\frac{E_{1}\left(T_{c}\right)}{T_{c}{ }^{2}} \delta T+\left(\frac{1}{T_{c}}+\left.\frac{1}{\hbar} \frac{d S}{d E}\right|_{E=E_{1}\left(T_{c}\right)}\right) \frac{d E_{1}}{d T} \delta T .
\end{gathered}
$$

Taking into account that at the point $E_{1}$

$$
\left.\frac{d f_{T}}{d E}\right|_{E=E_{1}}=-\frac{1}{T_{c}}-\left.\frac{1}{\hbar} \frac{d S}{d E}\right|_{E=E_{1}}=0
$$

we obtain

$$
\frac{\delta S_{\text {quantum }}}{\hbar}=-\frac{E_{1}}{T_{c}^{2}} \delta T
$$

Thus, the jump of the derivative at the point $T_{c}$ is

$$
\Delta \frac{d S_{\mathrm{Eucl}}}{d T}=\frac{\hbar\left(E_{1}-U_{0}\right)}{T_{c}{ }^{2}}<0,
$$

and the thermal action always decays more rapidly than the quantum one. We thus have shown that if the imaginary time oscillation time $\tau$ is not a monotonous function of energy $E$, a first-order transition takes place. Equation (13) quantifies the strength of the first-order transition: if $E_{1}\left(T_{c}\right) \simeq U_{0}$, the difference in the bounce solutions is small and 
the first-order transition is weak. In the opposite with case $E_{1}\left(T_{c}\right) \simeq 0$ the saddle-point solution deforms significantly and we have a strong first-order transition.

For a 1D massive particle the above physics can be easily realized. Consider a given function $\tau(E)$ for a trapped particle. Then there exist infinitely many potentials reproducing $\tau(E)$ as their oscillation times 13 . A simple way to obtain a non-monotonous behavior is to choose a function $\tau(E)$ with one minimum. For more complicated nonmonotonous dependences the system can exhibit several transitions. In general a system may exhibit several first-order transitions but not more than one of second-order.

All the results obtained in this section remain true for an arbitrary 1D metastable Hamiltonian system as Eq. (6) is applicable in this case.

\section{B. Nonlinear perturbation near the thermal saddle-point solution}

Consider a metastable system whose oscillation period in the imaginary time $\tau$ is a function of a scalar parameter $a$, $\tau=\tau(a)$. For Hamiltonian systems it is convenient to choose this parameter to be equal to the energy, i.e., $a=E$, see section II A. However, for dissipative systems one cannot use the energy as it is no more a conserved quantity. In this case we can parametrize the periodic imaginary time solutions of the equation of motion by the amplitude $a$ of the oscillations: The amplitude $a$ quantifies the difference between the thermal and the weakly time-dependent solutions in the vicinity of the thermal saddle-point solution (later we will show how to define such a notion as the "amplitude" for any metastable system). It can be easily understood that if $\tau(a)$ is not a monotonous function of the amplitude, the system exhibits a first-order transition: Starting from the zero temperature bounce solution the period $\tau=\hbar / T$ decreases with increasing temperature. However we cannot carry this solution beyond the temperature $\hbar / \tau_{\min }$ and thus will encounter a first-order jump to the thermal solution at some temperature $T_{c}<\hbar / \tau_{\min }$ (in the simplest case of a $\tau$-dependence with one minimum).

The basic idea leading to the criterion for a sharp transition from quantum to classical behavior is the following: Let us investigate the imaginary time oscillation period in the vicinity of the thermal saddle-point, where $a=0$. From the above discussion follows that if $\tau(a)-\tau(0)<0$, the system will exhibit a first-order transition. It turns out that a general expression for $\tau(a)$ can be obtained for a wide class of systems.

At high temperatures the saddle-point solution is time-independent. Let us slightly perturb this solution and calculate its oscillation time in the vicinity of the point $a=0$. We suppose that the imaginary time Lagrangian $L$ of the system under consideration can be written in the form

$$
L=T(\mathbf{u}, \dot{\mathbf{u}}, \mathbf{r})+V(\mathbf{u}, \mathbf{r}),
$$

where the term $T(\mathbf{u}, \dot{\mathbf{u}}, \mathbf{r})$ is responsible for the dynamical properties of the system and $V(\mathbf{u}, \mathbf{r})$ is the potential energy. The equation of motion corresponding to the Lagrangian (14) takes the form

$$
\hat{l} \mathbf{u}=\frac{\delta V}{\delta \mathbf{u}}
$$

Later we shall suppose that the operator $\hat{l}$ is linear and that $\delta \hat{l} / \delta \mathbf{r}=0$. This is not a strong restriction as the dynamical terms traditionally considered in the Lagrangian (14) consist of dissipative, massive, and Hall terms. Dissipation is usually described by the Caldeira-Leggett formalism 14 , leading to a linear term in the equation of motion. Massive and Hall terms also lead to linear equations, i.e., the operator $\hat{l}$ satisfies the conditions discussed above. For a massive particle the operator $\hat{l}$ takes the form $\hat{l}=m \partial^{2} / \partial t^{2}$.

In the high-temperature regime the solution of Eq. (15) $\mathbf{u}_{t h}(\mathbf{r})$ is time-independent. Let us expand Eq.(15) into a series around this solution. Substituting $\mathbf{u}(\mathbf{r}, t)=\mathbf{u}_{t h}(\mathbf{r})+\delta \mathbf{u}(\mathbf{r}, t)$ into Eq. (15) and expanding in $\delta \mathbf{u}$ we obtain

$$
\hat{l} \delta \mathbf{u}=\hat{h} \delta \mathbf{u}+\hat{G}_{2}[\delta \mathbf{u}]+\hat{G}_{3}[\delta \mathbf{u}],
$$

where $\hat{l}=\delta^{2} V / \delta \mathbf{u}^{2}$ is a linear operator and $\hat{G}_{2}$ and $\hat{G}_{3}$ are nonlinear operators satisfying the conditions

$$
\hat{G}_{2}[\lambda \mathbf{y}]=\lambda^{2} \hat{G}_{2}[\mathbf{y}] \quad \text { and } \quad \hat{G}_{3}[\lambda \mathbf{y}]=\lambda^{3} \hat{G}_{3}[\mathbf{y}],
$$

where $\mathbf{y}$ is an arbitrary vector and $\lambda$ is a constant. Our goal is to solve Eq. (16) for $\delta \mathbf{u}(\mathbf{r}, t)$ and find the correction to the oscillation period away from the thermal saddle point. To lowest order in perturbation theory we separate variables with the Ansatz

$$
\delta \mathbf{u}=a \mathbf{u}_{0}(\mathbf{r}) \cos \omega_{0} t
$$


and substituting (18) into (16) while neglecting terms of order higher than $a^{2}$ and higher we obtain

$$
\mathbf{u}_{0}(\mathbf{r})\left[\hat{l} \cos \omega_{0} t\right]=\left[\hat{h} \mathbf{u}_{0}(\mathbf{r})\right] \cos \omega_{0} t
$$

The function $\cos \omega_{0} t$ is an eigenfunction of the operator $\hat{l}, \hat{l} \cos \omega_{0} t=l\left(\omega_{0}\right) \cos \omega_{0} t$, and we obtain the eigenvalue equation for $\mathbf{u}_{0}(\mathbf{r})$

$$
l\left(\omega_{0}\right) \mathbf{u}_{0}(\mathbf{r})=\hat{h} \mathbf{u}_{0}(\mathbf{r}) .
$$

The operator $\hat{h}$ has one negative eigenvalue due to the unstable direction in phase space. On the other hand, $l\left(\omega_{0}\right)<0$, i.e., Eq. (20) has only one solution. The function $\mathbf{u}_{0}(\mathbf{r})$ then is the eigenfunction of the operator $\hat{h}$ corresponding to its lowest eigenvalue $h_{0}$ and the frequency $\omega_{0}$ is determined by the condition $l\left(\omega_{0}\right)=h_{0}$. Note that $T_{c}=\hbar \omega_{0} / 2 \pi$ is the transition temperature in case of a second-order transition. At this stage it becomes clear how to define such a notion as an "amplitude" for any tunneling problem: it is the expansion coefficient of the unstable mode.

Next, let us write

$$
\delta \mathbf{u}=a \mathbf{u}_{0}(\mathbf{r}) \cos \omega t+\delta \mathbf{u}_{2}(\mathbf{r}, t), \quad \omega=\omega_{0}+\omega_{2},
$$

where $\omega_{2}$ is the correction to the frequency $\omega_{0}$ and $\delta \mathbf{u}_{2} \sim a^{2}$. Substituting (21) into (16), neglecting terms of order $a^{3}$ and higher, and using (17) we obtain the equation for $\delta \mathbf{u}_{2}$

$$
l(\omega) a \mathbf{u}_{0}(\mathbf{r}) \cos \omega t+\hat{l} \delta \mathbf{u}_{2}=l\left(\omega_{0}\right) a \mathbf{u}_{0}(\mathbf{r}) \cos \omega t+\hat{h} \delta \mathbf{u}_{2}+a^{2} \hat{G}_{2}\left[\mathbf{u}_{0}(\mathbf{r})\right] \cos ^{2} \omega t .
$$

Rearranging terms, we arrive at

$$
\delta \mathbf{u}_{2}=(\hat{l}-\hat{h})^{-1}\left[\left(l\left(\omega_{0}\right)-l(\omega)\right) a \mathbf{u}_{0}(\mathbf{r}) \cos (\omega t)+\frac{a^{2}}{2} \hat{G}_{2}\left[\mathbf{u}_{0}(\mathbf{r})\right](\mathbf{1}+\cos (\mathbf{2} \omega \mathbf{t}))\right] .
$$

Since $(\hat{l}-\hat{h}) \mathbf{u}_{0}(\mathbf{r}) \cos \omega t=0$, the first term has to vanish and we obtain no shift in the frequency, $l(\omega)=l\left(\omega_{0}\right)$, i.e., $\omega=\omega_{0}, \omega_{2}=0$. The solution of Eq.(22) then reads

$$
\delta \mathbf{u}_{2}=\mathbf{g}_{1}(\mathbf{r})+\mathbf{g}_{2}(\mathbf{r}) \cos 2 \omega_{0} t
$$

where

$$
\begin{gathered}
\mathbf{g}_{1}(\mathbf{r})=-\frac{1}{2} a^{2} \hat{h}^{-1} \hat{G}_{2}\left[\mathbf{u}_{0}(\mathbf{r})\right] \\
\mathbf{g}_{2}(\mathbf{r})=-\frac{1}{2} a^{2}\left(\hat{h}-l\left(2 \omega_{0}\right)\right)^{-1} \hat{G}_{2}\left[\mathbf{u}_{0}(\mathbf{r})\right] .
\end{gathered}
$$

To third order in perturbation theory we make the Ansatz

$$
\delta \mathbf{u}=a \mathbf{u}_{0}(\mathbf{r}) \cos \omega t+\delta \mathbf{u}_{2}(\mathbf{r}, t)+\delta \mathbf{u}_{3}(\mathbf{r}, t), \quad \omega=\omega_{0}+\omega_{3} .
$$

Substituting (27) into (16) and neglecting terms of order $a^{4}$ and higher we obtain the equation for $\delta \mathbf{u}_{3}$,

$$
\begin{aligned}
& l(\omega) a \mathbf{u}_{0}(\mathbf{r}) \cos \omega t+l(2 \omega) \mathbf{g}_{2}(\mathbf{r}) \cos 2 \omega t+\hat{l} \delta \mathbf{u}_{3} \\
= & l\left(\omega_{0}\right) a \mathbf{u}_{0}(\mathbf{r}) \cos \omega t+\hat{h} \mathbf{g}_{1}+\hat{h} \mathbf{g}_{2} \cos 2 \omega t+\hat{h} \delta \mathbf{u}_{3} \\
+ & \hat{G}_{2}\left[a \mathbf{u}_{0}(\mathbf{r}) \cos \omega t+\mathbf{g}_{1}+\mathbf{g}_{2} \cos 2 \omega t\right]+\hat{G}_{3}\left[a \mathbf{u}_{0}(\mathbf{r}) \cos \omega t\right] .
\end{aligned}
$$

As $\mathbf{g}_{1}(\mathbf{r}), \mathbf{g}_{2}(\mathbf{r}) \sim a^{2}$, we can expand the term $\hat{G}_{2}\left[a \mathbf{u}_{0}(\mathbf{r}) \cos \omega t+\mathbf{g}_{1}+\mathbf{g}_{2} \cos 2 \omega t\right]$ around the function $a \mathbf{u}_{0}(\mathbf{r}) \cos \omega t$ :

$$
\begin{aligned}
& \hat{G}_{2}\left[a \mathbf{u}_{0}(\mathbf{r}) \cos \omega t+\mathbf{g}_{1}(\mathbf{r})+\mathbf{g}_{2}(\mathbf{r}) \cos 2 \omega t\right] \cong \\
& \hat{G}_{2}\left[a \mathbf{u}_{0}(\mathbf{r}) \cos \omega t\right]+\left.a \cos \omega t \frac{\delta \hat{G}_{2}}{\delta \mathbf{u}}\right|_{\mathbf{u}=\mathbf{u}_{0}(\mathbf{r})}\left(\mathbf{g}_{1}(\mathbf{r})+\mathbf{g}_{2}(\mathbf{r}) \cos 2 \omega t\right)
\end{aligned}
$$


Note that the opeator $\delta \hat{G}_{2} /\left.\delta \mathbf{u}\right|_{\mathbf{u}_{0}}$ is a linear operator, see Eq. (17).

As before we sum up all the resonant terms $\sim \cos \omega t \mathbf{u}_{0}(\mathbf{r})$ and obtain the following equation for the shift in the oscillation frequency

$$
l(\omega)-l\left(\omega_{0}\right)=\frac{a^{2}}{\left(\mathbf{u}_{0}, \mathbf{u}_{0}\right)}\left(\mathbf{u}_{0}, \mathbf{f}\left[u_{0}(\mathbf{r})\right]\right)
$$

where

$$
\mathbf{f}\left[u_{0}(\mathbf{r})\right]=-\left.\frac{1}{2} \frac{\delta \hat{G}_{2}}{\delta \mathbf{u}}\right|_{\mathbf{u}=\mathbf{u}_{0}(\mathbf{r})}\left[\hat{h}^{-1}+\frac{1}{2}\left(\hat{h}-l\left(2 \omega_{0}\right)\right)^{-1}\right] \hat{G}_{2}\left[\mathbf{u}_{0}(\mathbf{r})\right]+\frac{3}{4} \hat{G}_{3}\left[\mathbf{u}_{0}(\mathbf{r})\right]
$$

and $(\mathbf{x}, \mathbf{y})$ denotes a scalar product (see Appendix for a general discussion). The criterion for a first-order transition reads $\tau(a)-\tau(0)<0$ and using $d l / d \omega<0$ we find

$$
\left(\mathbf{u}_{0}(\mathbf{r}), \mathbf{f}\left[u_{0}(\mathbf{r})\right]\right)<0 .
$$

In the next section we apply the criterion (32) to the tunneling of strings.

\section{TUNNELING OF STRINGS}

\section{A. General theory}

In this section we apply Eqs. (31) and (32) to the problem of a driven string $i$ ) tunneling between two potential wells and $i$ ) depinning from a columnar defect. Within the linear elasticity theory the imaginary time Lagrangian of the string takes the form

$$
\mathcal{L}=\int_{-L / 2}^{L / 2} d z\left(L_{D}\left(u, \partial_{t} u\right)+\frac{\epsilon}{2}\left(\frac{\partial u}{\partial z}\right)^{2}+V(u)-F u\right)
$$

Below we consider the dynamical term $L_{D}\left(u, \partial_{t} u\right)$ to be the sum of massive and dissipative terms (we ignore the transverse contribution from the Hall term)

$$
L_{D}\left(u, \partial_{t} u\right)=\frac{\rho}{2}\left(\partial_{t} u\right)^{2}-\frac{\eta}{2 \pi} \partial_{t} u \int_{-\hbar / 2 T}^{+\hbar / 2 T} d t_{1} \ln \left|\sin \left[\pi\left(t-t_{1}\right) \frac{T}{\hbar}\right]\right| \partial_{t_{1}} u .
$$

$L$ and $\epsilon$ are, respectively, the length and the elasticity of the string, $V(u)$ is the potential, the external force is assumed to be small: $F \ll F_{c}$ with $F_{c}$ the depinning force. The thermal saddle-point solution $u_{t h}(\mathbf{r})$ corresponding to the Lagrangian satisfies the equation

$$
\epsilon \frac{\partial^{2} u}{\partial z^{2}}=\frac{\partial V}{\partial u}-F
$$

The operators $\hat{h}, \hat{G}_{2}, \hat{G}_{3}$, and $\delta \hat{G}_{2} /\left.\delta u\right|_{u_{0}(z)}$ take the following form,

$$
\begin{gathered}
\hat{h}=-\epsilon \frac{\partial^{2}}{\partial z^{2}}+\left.\frac{\partial^{2} V}{\partial u^{2}}\right|_{u_{t h}(z)}, \\
\hat{G}_{2}[y]=\left.\frac{1}{2} \frac{\partial^{3} V}{\partial u^{3}}\right|_{u_{t h}} y^{2}, \quad \hat{G}_{3}[y]=\left.\frac{1}{6} \frac{\partial^{4} V}{\partial u^{4}}\right|_{u_{t h}} y^{3},\left.\quad \frac{\delta \hat{G}_{2}}{\delta u}\right|_{u_{0}}(y)=\left.\frac{\partial^{3} V}{\partial u^{3}}\right|_{u_{t h}} u_{0}(z) y,
\end{gathered}
$$

where $y$ is an arbitrary function of $z$. The scalar product $\left(y_{1}, y_{2}\right)$ of two arbitrary functions $y_{1}(z)$ and $y_{2}(z)$ is defined as $\int_{-L / 2}^{+L / 2} y_{1}(z) y_{2}(z) d z$.

Eq. (32) takes the form 


$$
\begin{aligned}
& \left(u_{0}, f\left[u_{0}\right]\right)=-\left.\left.\frac{1}{4} \int_{-L / 2}^{L / 2} d z \frac{\partial^{3} V}{\partial u^{3}}\right|_{u_{t h}} u_{0}^{2}\left(\hat{h}^{-1}+\frac{1}{2}\left(\hat{h}-l\left(2 \omega_{0}\right)\right)^{-1}\right) \frac{\partial^{3} V}{\partial u^{3}}\right|_{u_{t h}} u_{0}^{2} \\
& +\left.\frac{1}{8} \int_{-L / 2}^{L / 2} d z \frac{\partial^{4} V}{\partial u^{4}}\right|_{u_{t h}} u_{0}^{4}<0,
\end{aligned}
$$

where $l(w)=-\rho \omega^{2}-\eta|\omega|$. It is appropriate to mention that the operator $\hat{h}$ has one zero eigenvalue in the limit $L \rightarrow \infty$, (see III B) such that taking its inverse $\hat{h}^{-1}$ needs some care: The eigenfunction corresponding to the zero eigenvalue is an odd function of $z$, whereas $u_{0}^{2} \partial^{3} V /\left.\partial u^{3}\right|_{u_{t h}}$ is an even function and there is no contribution to the integral in Eq. (38). Consequently, the contribution of the eigenfunction corresponding to the zero eigenvalue is equal to zero.

Let us investigate the sign of Eq. (38) in the two cases mentioned above.

\section{B. Motion of a string across a slightly tilted periodic potential}

The tunneling of a string across a titled periodic potential is a convenient model for the description of the motion of dislocations across the Peierls barrier. In the case being studied $V(u)$ is the periodic part of the potential (see Eq. (33)). Below we denote time by $t$ and the oscillation period by $\tau$. This problem shows a smooth crossover from a purely thermal to a thermal-assisted quantum activation at a temperature $T_{c}=T_{0}$ of order $\hbar(F / \rho d)^{1 / 2}$ (see Ref. B) with $d$ the period of the potential $V(u)$. The schematic evolution of the nucleus is shown in Figs. 5(a)-5(c). At temperatures smaller than $T_{1} \sim \hbar F /\left(\rho V_{0}\right)^{1 / 2}$, with $V_{0}$ the amplitude of the potenrial, the nucleus is of circular shape (see Fig. 5(a))

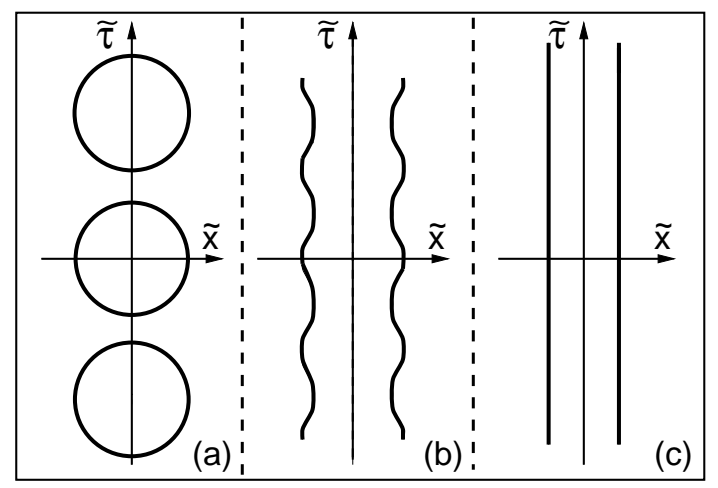

Fig.5 Evolution of the nucleus (in coordinates $\tilde{z}=z / \sqrt{\epsilon}$ and $\tilde{t}=t / \sqrt{\rho}$ ). (a) Nucleus at low temperatures. (b) Nucleus at intermediate temperatures where the interaction between the nucleus' walls is relevant. (c) Nucleus at high temperatures. The string overcomes the barrier due to thermal activation.

with the bounce solution $u(z, t) \cong a \Theta(R-r), R \sim \sqrt{V_{0}} / F$ is the radius of the nucleus $\left(r^{2}=z^{2} / \epsilon+t^{2} / \rho \equiv \tilde{z}^{2}+\tilde{t}^{2}\right)$ and the Euclidean action $S \sim \sqrt{\rho \epsilon} d V_{0} / F$. At $T>T_{1}$ we have to take into account the interaction of the nucleus' walls (Fig. 5(b)). At $T>T_{c}=T_{0}$ the string overcomes the barrier due to thermal activation with $U \sim d \sqrt{\epsilon V_{0}}$ (Fig. 5(c)). The thermal solution then can be obtained by a continuous deformation of the zero temperature bounce. As the problem exhibits a second-order transition from quantum to classical behavior, we should expect $l(\omega)-l\left(\omega_{0}\right)>0$. Let us prove this inequality. The "potential" in the one-dimensional Schrödinger operator $\hat{h}$ (see (36)) is shown in Fig. 6. The spectrum of $\hat{h}$ consists of one negative, one zero (in the limit $L \rightarrow \infty$ ), and positive eigenvalues (one can easily verify that the function $\partial u_{t h} / \partial z$ is an eigenfunction of the operator $\hat{h}$ with zero eigenvalue; on the other hand, $\partial u_{t h} / \partial z$ has one node, i.e., there is one eigenfunction with a negative eigenvalue). In the limit $F \rightarrow 0$ the negative eigenvalue $h_{0}$ tends to zero as $-F$, see Ref. of order $V_{0} / d^{2}$. Analysing the terms in Eq. (38) we note that the operator $\left[\hat{h}^{-1}+1 / 2\left(\hat{h}-l\left(2 \omega_{0}\right)\right)^{-1}\right]$ has a large 
negative eigenvalue $\sim-1 / F$ originating from the unstable direction in the phase space. The remaining spectrum is positive and does not depend on $F$, as is also the case for the other factors appearing in Eq. (38).

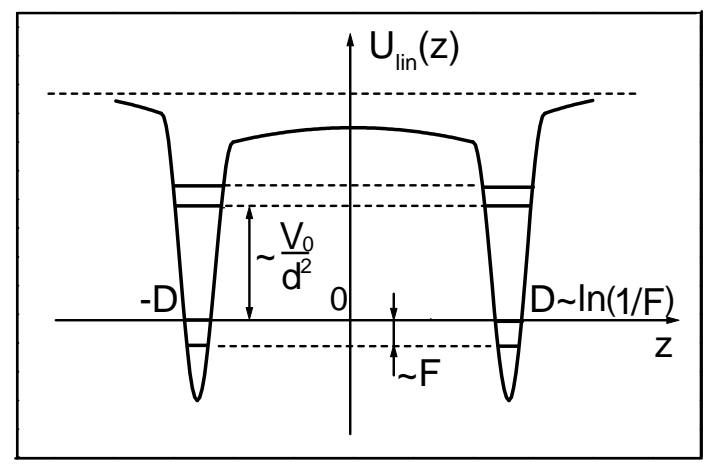

Fig.6 The potential in the 1D Schrödinger operator for the linearized problem of a string tunneling of a string across a slightly titled periodic potential. $U_{\text {lin }}(z)=\partial^{2} U /\left.\partial z^{2}\right|_{u_{t h}(z)}$. The negative eigenvalue appears because of the interaction of the two wells. The distance between the negative and zero eigenvalues is much smaller than the distance between possible positive eigenvalues of the discrete spectrum and the zero-eigenvalue level.

Thus we can approximate $\left(u_{0}, f\left[u_{0}\right]\right)$ by the contribution from the unstable direction and arrive at the estimate

$$
\left(u_{0}, f\left[u_{0}\right]\right) \simeq-\frac{1}{4}\left(\frac{1}{h_{0}}+\frac{1}{2\left(h_{0}-l\left(2 \omega_{0}\right)\right)}\right)\left(\int_{-L / 2}^{L / 2} \frac{\partial^{3} V}{\partial u^{3}} u_{0}^{3} d z\right)^{2}
$$

The second term in Eq. (38) does not involve the small parameter $F$ and can always be neglected in the limit $F \rightarrow 0$. As $l(\omega)=-\rho \omega^{2}-\eta|\omega|, h_{0}=l\left(\omega_{0}\right)$ we obtain

$$
\frac{1}{h_{0}}+\frac{1}{2\left(h_{0}-l\left(2 \omega_{0}\right)\right)}<0
$$

and, consequently, $l(\omega)-l\left(\omega_{0}\right)>0$. We have shown therefore that in the vicinity of the point $a=0, \tau(a)$ is an increasing function of $a$. This is in agreement with the fact that the problem of the tunneling of a massive string across a slightly titled periodic potential exhibits a second-order transition 3 .

\section{Depinning of a string from a linear defect}

The behavior described above should be contrasted with the sharp transition obtained recently for the tunneling of a massive string from a linear defect 15 . The imaginary time Lagrangian has the form $(33)(\eta=0)$ with the potential $U(u)$ describing a single potential well of depth $V_{0}$ and radius $d$. At low temperatures the nucleus has a circular shape as well. The macroscopic part of the bounce solution can be written in the form

$$
u(z, t)=-\frac{F}{4} r^{2}+\frac{2 V_{0}}{F}, \quad r^{2}=\frac{z^{2}}{\epsilon}+\frac{t^{2}}{\rho} \equiv \tilde{z}^{2}+\tilde{t}^{2} .
$$

The substitution of Eq. (41) into Eq. (33) gives the Euclidean action

$$
\frac{S_{q}}{\hbar}=\frac{1}{\hbar} \int L[u(z, t)] d t=\frac{4 \pi}{\hbar} \sqrt{\rho \epsilon}\left(\frac{V_{0}}{F}\right)^{2} .
$$

The thermal exponent is given by $U / T=(4 \sqrt{2} / 3) \sqrt{\epsilon V_{0}}\left(V_{0} / F T\right) . S_{q} / \hbar$ and $U / T$ become equal at $T_{c}=$ $(\sqrt{2} / 3 \pi) \hbar F /\left(\rho V_{0}\right)^{1 / 2}$. On the other hand, the dynamical solution (41) remains valid as long as the radius of the nucleus is smaller than the periodicity in $t$, i.e., for $T<T_{1}=3 \pi / 8 T_{c}$. The formal application of the perturbative procedure (see section IIB gives the following linearized problem determining the crossover temperature $T_{0}$, 


$$
-\epsilon \frac{\partial^{2} u_{0}}{\partial z^{2}}+\left.\frac{\partial^{2} V}{\partial u^{2}}\right|_{u_{t h}(z)} u_{0}=-\rho\left(\frac{2 \pi T_{0}}{\hbar}\right)^{2} u_{0} .
$$

The 1D Schrödinger operator in (43) has again one negative eigenvalue which does not depend on the details of the potential $V(u)$ in the limit $F \rightarrow 0$ (Ref.5 5 (16). $T_{0}$ then is givep by the formula $T_{0}=\left(\mu / 2^{3 / 2} \pi\right) \hbar F / \sqrt{\rho V_{0}}=0.900 T_{c}$ with $\mu=1.199$ the root of the equation $\mu \tanh \mu=1$, see Ref 16 . As $T_{0}<T_{c}<T_{1}$, the string exhibits a first-order transition at the temperature $T_{c}$. In this case the quantum solution (see Fig. 5(a)) jumps at $T_{c}$ to the thermal solution (Fig. 5(c)). The intermediate regime (Fig. 5(b)) is absent.

This sharp transition is again in agreement with the above considerations: There exist solutions of the equation of motion in imaginary time with periods $\tau \in\left(\hbar / T_{1}, \infty\right)$ and energy close to zero (we consider a massive dynamics here). On the other hand, at the temperature $T_{0}$ there appears a time-dependent solution of the equation of motion with a period $\hbar / T_{0}$ and an energy equal to the energy of the thermal solution $U$. As $\hbar / T_{1}<\hbar / T_{0}$, the dependence of the oscillation period on energy is not monotonous and a first-order transition takes place. In the present case the jump of the derivative $\left[E_{1}\left(T_{c}\right)-U\right] / T_{c}{ }^{2}$ in the action is large: $E_{1}\left(T_{c}\right)$ is close to zero, $U$ and $T_{c}^{-1}$ are proportional to $1 / F$, hence $\Delta\left(\partial S_{\text {Eucl }} / \partial T\right) \sim-1 / F^{3}$ and one can see that we deal with a strong first-order transition.

Let us find the sign of $l(\omega)-l\left(\omega_{0}\right)$ in $(30)$ for the case $\eta \neq 0, \rho \neq 0$. The "potential" in the one-dimensional Schrödinger operator $\hat{h}$ is shown in Fig. 7 .

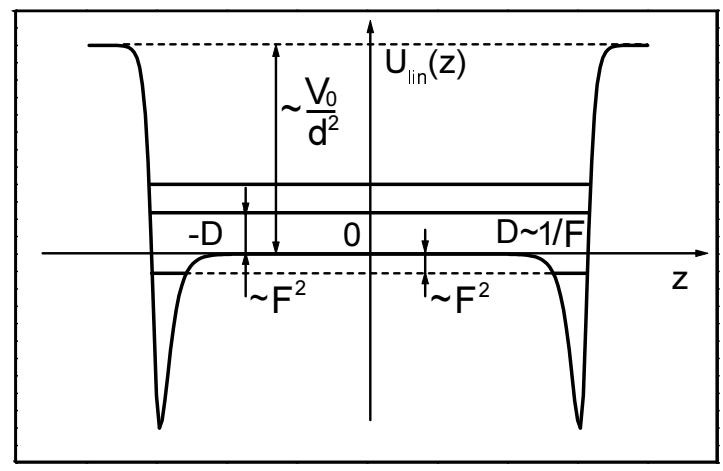

Fig.7 The potential in the 1D Schrödinger operator for the linearized problem of the depinning of a string from a linear defect. $U_{l i n}(z)=\partial^{2} U /\left.\partial z^{2}\right|_{u_{t h}(z)}$. The modulus of the negative eigenvalue is of the same order as positive eigenvalues in the discrete spectrum.

In the limit $F \rightarrow 0$ the most significant contribution to the integrals in (38) arises from the region $|z|>D=\sqrt{2 \epsilon U_{0}} / F$. The spectrum of the operator $\hat{h}$ consists of one negative, one zero, and positive eigenvalues. In the limit $F \rightarrow 0$ the negative eigenvalue and the lowest positive eigenvalues of the descrete spectrum are proportional to $F^{2}$ and we need to analyze the first integral in Eq. (38) more carefully than before. The second integral in Eq. (38) is again irrelevant. In the limit $F \rightarrow 0$, the eigenfunctions of the lowest levels take the same form in the region $|z|>D$, i.e., we can write

$$
\begin{aligned}
\left(u_{0}, f\left[u_{0}\right]\right) & \cong-\left.\left.\frac{1}{4} \int_{-L / 2}^{L / 2} d z \frac{\partial^{3} V}{\partial u^{3}}\right|_{u_{t h}} u_{0}^{2}\left(\hat{h}^{-1}+\frac{1}{2}\left(\hat{h}-l\left(2 \omega_{0}\right)\right)^{-1}\right) \frac{\partial^{3} V}{\partial u^{3}}\right|_{u_{t h}} u_{0}^{2} \\
& =-\left.\left.\frac{1}{4} \int_{-L / 2}^{L / 2} d z \frac{\partial^{3} V}{\partial u^{3}}\right|_{u_{t h}} u_{0}^{2}\left(\sum_{n}\left(h_{n}^{-1}+\frac{1}{2}\left(h_{n}-l\left(2 \omega_{0}\right)\right)^{-1}\right)\left|u_{n}\right\rangle\left\langle u_{n}\right|\right) \frac{\partial^{3} V}{\partial u^{3}}\right|_{u_{t h}} u_{0}^{2} \\
& \cong-\frac{1}{4} \sum_{n}\left(h_{n}^{-1}+\frac{1}{2}\left(h_{n}-l\left(2 \omega_{0}\right)\right)^{-1}\right)\left(\left.\int_{|z|>D} d z u_{n} \frac{\partial^{3} V}{\partial u^{3}}\right|_{u_{t h}} u_{0}^{2}\right)^{2} .
\end{aligned}
$$

Here, we have used that $\partial^{3} V /\left.\partial u^{3}\right|_{u_{t h}} \simeq 0$ for $|z|<0$. In the region $|z|>D, u_{n}= \pm C_{n} u_{0} / C_{0}$ with $C_{n}$ denoting the normalizers. Consequently, 


$$
\left(u_{0}, f\left[u_{0}\right]\right) \cong-\frac{1}{4}\left(\left.\int_{|z|>D} \frac{\partial^{3} V}{\partial u^{3}}\right|_{u_{t h}} u_{0}^{3} d z\right)^{2} \sum_{n} C_{n}^{2}\left(h_{n}{ }^{-1}+\frac{1}{2}\left(\hat{h}_{n}-l\left(2 \omega_{0}\right)\right)^{-1}\right) .
$$

For the lowest eigenvalue we have $u_{0}(|z|<D)=C_{0} \cosh \left(\sqrt{\frac{\left|h_{0}\right|}{\epsilon} z}\right)$ and the eigenfunction belonging to the zero eigenvalue takes the form $u_{1}(|z|<D)=C_{1} z$. In the limit $F \rightarrow 0$ we have $u_{n}^{\prime} /\left.u_{n}\right|_{z=D^{-}}=u_{n}^{\prime} /\left.u_{n}\right|_{z=D^{+}}=u_{0}^{\prime} /\left.u_{0}\right|_{z=D^{+}}$, i.e., $u_{n}^{\prime} /\left.u_{n}\right|_{z=D^{-}}=u_{0}^{\prime} /\left.u_{0}\right|_{z=D^{-}}$(we use the condition of continuity of the logarithm derivative), i.e., for the lowest eigenvalue we obtain the condition

$$
\sqrt{\frac{\left|h_{0}\right|}{\epsilon}} D \tanh \left(\sqrt{\frac{\left|h_{0}\right|}{\epsilon}} D\right)=1
$$

Only even levels contribute to the integral in (38) and their eigenfunctions are given by $C_{n} \cos \left(\sqrt{\frac{h_{n}}{\epsilon}} z\right)$, hence we obtain for $h_{n}$

$$
\sqrt{\frac{h_{n}}{\epsilon}} D \tan \left(\sqrt{\frac{h_{n}}{\epsilon}} D\right)=-1
$$

The most significant contribution to the integrals $\int_{-L / 2}^{L / 2} u_{n}^{2} d z$ arises from the interval $|z|<D$, i.e.,

$$
C_{0}^{2}=\frac{1}{\int_{-D}^{D} \cosh ^{2}\left(\sqrt{\frac{\left|h_{o}\right|}{\epsilon}} z\right) d z}=\frac{1}{D \cosh ^{2}\left(\sqrt{\frac{\left|h_{o}\right|}{\epsilon}} D\right)}
$$

and

$$
C_{n}^{2}=\frac{1}{\int_{-D}^{D} \cos ^{2}\left(\sqrt{\frac{h_{n}}{\epsilon}} z\right) d z}=\frac{1}{D \cos ^{2}\left(\sqrt{\frac{h_{n}}{\epsilon}} D\right)}, \quad n=2,4 \ldots
$$

In Eqs. (48) and (49) we have used Eqs. (46) and (47).

Let us suppose that $\eta=0$. Later we will show that if the problem exhibits a first-order transition for $\eta=0$, the kind of transition remains the same for any $\eta \neq 0$. Numerical solution of Eqs. (46) and (47) show that $h_{0}=$ $-1.439229 \epsilon / D^{2}$ and $h_{2}=7.830964 \epsilon / D^{2}$. For the case of a purely massive dynamics $l\left(2 \omega_{0}\right)=4 l\left(\omega_{0}\right)=4 h_{0}$. The operator $\hat{h}^{-1}+(1 / 2)\left(\hat{h}-l\left(2 \omega_{0}\right)\right)^{-1}$ has only one negative eigenvalue, i.e., if we show that the sum

$$
S=\sum_{n}^{M} C_{n}^{2}\left(\frac{1}{h_{n}}+\frac{1}{2\left(h_{n}-l\left(2 \omega_{0}\right)\right)}\right), \quad n=0,2 \ldots
$$

is positive for some finite $M$, a first-order transition takes place. With $M=2$ we obtain

$$
C_{0}^{2}\left(\frac{1}{h_{0}}+\frac{1}{2\left(h_{0}-l\left(2 \omega_{0}\right)\right)}\right)+C_{2}^{2}\left(\frac{1}{h_{2}}+\frac{1}{2\left(h_{2}-l\left(2 \omega_{0}\right)\right)}\right)=0.008796 D / \epsilon>0,
$$

i.e., the transition from quantum to classical behavior is of first-order.

Finally, let us show that the kind of transition remains the same for any $\eta \neq 0$. We can write for the ratio

$$
\frac{l\left(2 \omega_{0}\right)}{l\left(\omega_{0}\right)}=\frac{4 \rho \omega_{0}^{2}+2 \eta \omega_{0}}{\rho \omega_{0}^{2}+\eta \omega_{0}}=4-\frac{2 \eta \omega_{0}}{\rho \omega_{0}^{2}+\eta \omega_{0}}<4,
$$

i.e., the relative contribution of the operator $(1 / 2)\left(\hat{h}-l\left(2 \omega_{0}\right)\right)^{-1}$ becomes even larger compared to the contribution of the operator $\hat{h}^{-1}$.

Comparing the problems considered in sections IIIB and III G the main difference appears because of the different character of the spectrum of the linearized problem. For the case of a string tunneling across a slightly titled periodic potential the most significant contribution arises from the negative eigenvalue. The contributions of the positive eigenvalues are negligible in the limit $F \rightarrow 0$. On the other hand, for a string depinning from a linear defect the contributions of the positive eigenvalues are of the same order as that of the negative eigenvalue. 


\section{CONCLUSION}

If a second order transition takes place, it is possible to use a perturbative approach for the calculation of the crossover temperature. In this case we have to substitute equation (1) into the equation of motion. Close to $T_{c}$ the function $\delta \mathbf{u}(\mathbf{r})$ is small and we obtain the linearized eigenvalue problem (see also Eq. (20))

$$
\left.\frac{\delta^{2} S_{\mathrm{Eucl}}[\mathbf{u}]}{\delta \mathbf{u}^{2}}\right|_{\mathbf{u}=\mathbf{u}_{0}(\mathbf{r})} \delta \mathbf{u}=\lambda \delta \mathbf{u}
$$

where the only negative eigenvalue of Eq. (53) (due to the single unstable direction in the phase space) determines the transition temperature $T_{c}$. For the case of a first order transition at $T_{c}$ the formal application of the perturbative procedure again gives some "crossover" temperature $T_{0}$ which comes to lie below the true transition at $T_{c}$, however. For the dependence of the oscillation time on energy considered above (see Fig. 4) $T_{0}=\hbar / \tau_{0}$. In Fig. 8 we plot the actions for the various extremal solutions corresponding to the situation in Fig. 4.

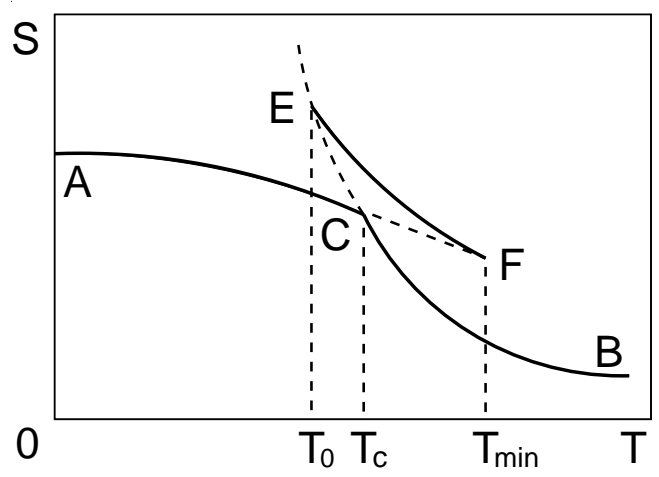

Fig.8 Euclidean action $S$ as a function of temperature $T$ for a non-monotonous oscillation time $\tau(E)$ as given in Fig. 4 .

The solid line $A C$ corresponds to quantum tunneling. At the point $C$, a first-order transition takes place and the solid line $C B$ shows the thermal action. In the point $E$ the "false" crossover takes place. The line $E F$ corresponds to the oscillating motion of the tunneling system with energy $E \in\left(E_{\min }, U_{0}\right)$ (see Fig. 4). The dashed line $C F$ is obtained by the continuation of the quantum action into the region $T>T_{c}$ and corresponds to the motion with energy $E \in\left(E_{1}\left(T_{c}\right), E_{\min }\right)$. In the point $F$ this line and the "false" crossover action intersecteach other. The dashed line $E C$ is a formal continuation of the thermal action into the region $T<T_{c}$, see also Ref.8.

The above discussion shows that in the case of a first-order transition one cannot use the perturbative representation of the bounce solution (see equation (11)). The transition takes place at a temperature higher than that given by the formal solution of the linearized eigenvalue problem. In this case one has to find the low temperature expression for the Euclidean action and determine the point where it is equal to the thermal action.

There are a number of articles concerning the decay of metastable states, where a sharp transition from quantum to classical behavior has been discovered. A first-order transition has been obtained by Lifshitz and Kagan in Ref. concerning phase transitions at low temperatures, by Chudnovsky 8 for a massive particle in a $1 \mathrm{D}$ potential for the case of a nonmonotonous dependence of the imaginary time oscillation period on energy, by Morais-Smith, Ivlev, and Blatter in Ref.17 where macroscopic quantum tunneling in a dc SQUID has been inyestigated, by Carriga in Ref. . $^{-17}$ concerning the vacuum decayin 2 and 3 dimensions in the thin wall limit, by Ferrera 19 for a bubble nucleation in the $\phi^{4}$ model, and by Skvortsov 15 for the case of the depinning of a massive string from a linear defect.

It would be interesting to observe a first-order transition in an experiment. Measurements of the escape rate of Josephson junctions from their zero voltage state20 show that the transition from quantum to classical behavior is of the second kind. The criterion described above allows us to find metastable systems exhibiting a sharp transition without a complicated solution of the equation of motion in the whole temperature range. The problem considered in section III of the depinning of a string with finite mass and friction coefficients is related to the problem of the depinning of vortices from columnar defects produced by the irradiation of heavy ions in high- $T_{c}$ superconductors. As it has been shown, this problem exhibits a first-order transition. In principle, this sharp behavior can be observed in an experiment. However, one should take into account that the point of transition is smeared due to the different radii of the defects. Hence, samples with defects of one radius should be taken for an experiment. An appropriate method to produce defects with identical radii is based on lithographic technique, see Ref.21 24. 
Finally, let us discuss the $F-T$ "phase diagram" of a massive string depinning from a columnar defect. At small $F, T_{c}$ increases $\sim F$ and the transition is first-order like. At $F \lesssim F_{c}$, with $F_{c}$ the critical force, the problem exhibits a secgnd-order transition with $T_{c}=T_{0} \sim \sqrt{F_{c}-F}$, see Ref.25. Consequently, there is a "tricritical" point $C$ (see also Ref.5), where the nature of the transition changes, see Fig. 9.

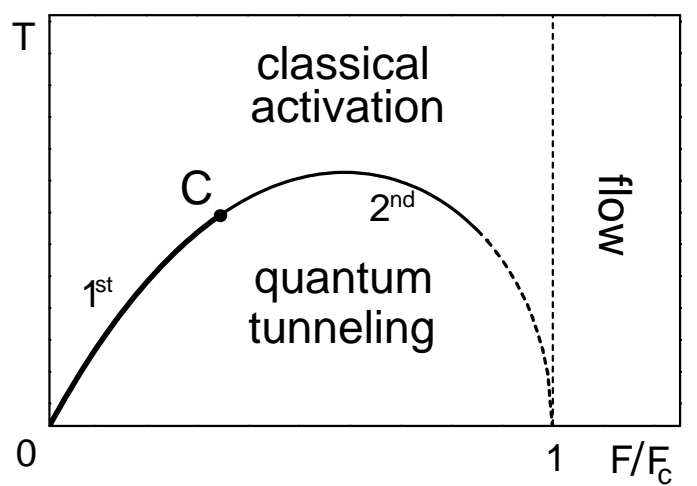

Fig.9 Possible $F-T$ "phase-diagram" of a massive string depinning from a columnar defect. In $C$ the kind of transition changes from first- to second-order, i.e., it is a tricritical point. In the vicinity of the point $F / F_{c}=1$ the curve is dashed as in the limit $F \rightarrow F_{c}$ the barrier disappears and the semiclassical approximation is not applicable.

In the vicinity of the point $F / F_{c}=1$ the transition curve is shown to be dashed as in the limit $F \rightarrow F_{c}$ the potential barrier disappears and the semiclassical approximation is not applicable.

As it has already been mentioned, the kind of transition is determined by the "competition" of the negative and positive eigenvalues of the operator $\left(\hat{h}^{-1}+(1 / 2)\left(\hat{h}-l\left(2 \omega_{0}\right)\right)^{-1}\right)$, see section III B. Let us investigate possible spectra of this operator for the case of the tunneling of a string. Consider a 1D Schrödinger operator $\hat{h}=-\epsilon \partial^{2} / \partial z^{2}+U_{\text {lin }}(z)$ of the linearized problem, where the function $U_{\text {lin }}(z)$ is such that the operator $\hat{h}$ has one negative and one zero eigenvalue. Let us try to reconstruct the initial metastable potential $V(u)$. As it has been mentioned in section IIIB, the function $\Psi=\partial u_{t h} / \partial z$ is a solution of the equation $\hat{h} \Psi=0$, i.e., $u_{t h}(z)$ satisfies the equation

$$
\frac{\epsilon}{2}\left(\frac{\partial u_{t h}}{\partial z}\right)^{2}-V\left(u_{t h}(z)\right)=0
$$

where we used the boundary condition $u_{t h}, u_{t h}^{\prime} \rightarrow 0$ if $|z| \rightarrow \infty$ and $V(0), V^{\prime}(0)=0$. Inverting the equation $u=$ $u_{t h}(z) \rightarrow z=u_{t h}^{-1}(u)$ we obtain

$$
U(u)=\left.\frac{\epsilon}{2}\left(\frac{\partial u_{t h}(z)}{\partial z}\right)^{2}\right|_{z=u_{t h}^{-1}(u)} .
$$

Hence, we have shown that for an arbitrary 1D Schrödinger operator of the linearized problem there is only one corresponding "original" metastable potential $V(u)$. We have shown therefore that the mapping $V(u) \rightarrow \hat{h}$ is one-toone. Consequently, one can construct a potential $V(u)$ such that the linearized "quantum mechanical" problem has the appropriate spectrum. Let the potential $V(u)$ be dependent on some parameter $\alpha$ which might be multicomponent, in general. Changing this parameter we are modifying the spectrum of the operator $\left(\hat{h}^{-1}+(1 / 2)\left(\hat{h}-l\left(2 \omega_{0}\right)\right)^{-1}\right)$ and thus we can tune the kind of the transition from quantum to classical behavior. In general the "phase diagram" might be more complicated than that in Fig. 9.

Briefly summarizing, we have studied the behavior of the imaginary time oscillation period of a metastable system in the vicinity of the saddle-point solution and have derived a sufficient criterion for a sharp first-order transition from quantum to thermal decay of a metastable state. The results have been applied to a comparative study of the tunneling of a massive string across a slightly titled periodic potential and the depinning of a string from a columnar defect in the presence of arbitrarily strong dissipation. The former problem shows a positive derivative of the oscillation period with respect to the amplitude of the motion, in agreement with the second-order transition for a purely massive problem 3 , whereas the latter problem shows a sharp behavior for any mass and friction coefficient. 


\section{ACKNOWLEDGMENTS}

We thank M.A. Skvortsov for helpful discussions and for sending us his results before publication. One of us (D.A.G.) acknowleges financial support from the Swiss National Foundation.

\section{APPENDIX A: CONDITION OF DISAPPEARENCE OF RESONANT TERMS}

Consider the following equation

$$
\hat{l} \mathbf{y}=\hat{h} \mathbf{y}+\mathbf{s}(\mathbf{r}, t)
$$

where the operators $\hat{l}$ and $\hat{h}$ act on time and spatial variables, respectively, $\mathbf{s}(\mathbf{r}, t)$ is a given vector. The spectrum of the operator $\hat{l}$ is negative and the spectrum of $\hat{h}$ shall be nonnegative except for one eigenvalue. The solution of Eq. (A1) takes the form

$$
\mathbf{y}=(\hat{l}-\hat{h})^{-1} \mathbf{s}(\mathbf{r}, t)
$$

As $[\hat{l}, \hat{h}]=0$, the eigenfunctions of the operator $(\hat{l}-\hat{h})$ are products of the eigenfunctions of the operators $\hat{l}$ and $\hat{h}$, respectively. The operator $(\hat{l}-\hat{h})$ might have one zero eigenvalue in which case its inverse $(\hat{l}-\hat{h})^{-1}$ is not properly defined. One then can use Eq. (A2) only if the coefficient of the zero eigenvalue eigenfunction of the operator $(\hat{l}-\hat{h})$ in the expansion of $\mathbf{s}(\mathbf{r}, t)$ over the eigenfunctions of the operator $(\hat{l}-\hat{h})$ is equal to zero, i.e., if

$$
\left(\mathbf{s}, \mathbf{V}_{0}\right)=0
$$

where $(\mathbf{x}, \mathbf{y})$ is a scalar product and $\mathbf{V}_{0}$ is the eigenfunction of the operator $(\hat{l}-\hat{h})$ corresponding to the zero eigenvalue.

${ }^{1}$ H.A. Kramers, Physica 7, 284 (1940).

${ }^{2}$ B.V. Petukhov and V.L. Pokrovsky, Zh. Eksp. Teor. Fiz. 63, 634 (1972) [Sov. Phys. JETP 36, 336 (1973)].

${ }^{3}$ B.I. Ivlev and V.I. Mel'nikov, Phys. Rev. B 36, 6889 (1987).

${ }^{4}$ A.I. Larkin and Yu.N. Ovchinnikov, Phys. Rev. B 28, 6281 (1983).

${ }^{5}$ for a review see G. Blatter, M.V. Feigel'man, V.B. Geshkenbein, A.I. Larkin, and V.M. Vinokur, Rev. Mod. Phys. 66, 1125 (1994).

${ }^{6}$ A.I. Larkin and Yu.N. Ovchinnikov, Pis'ma Zh. Eksp. Teor. Fiz. 37, 322 (1983) [JETP Lett. 37, 382 (1983)].

${ }^{7}$ I.M. Lifshitz and Yu. Kagan, Zh. Eksp. Teor. Fiz. 62, 1 (1972) [Sov. Phys. JETP 35, 206 (1972)].

${ }^{8}$ E. M. Chudnovsky, Phys. Rev. A 46, 8011 (1992).

${ }^{9}$ V.M. Galitsky, B.M. Karnakov, and V.I. Kogan, Zadachi po kvantowoi mekhanike, [in Russian (Problems in Quantum Mechanics)] (Nauka, Moscow, 1992).

${ }^{10}$ A.I. Larkin and Yu.N. Ovchinnikov, Zh. Eksp. Teor. Fiz. 86, 719 (1984) [Sov. Phys. JETP 59, 420 (1984)].

${ }^{11}$ V.I. Mel'nikov, Zh. Eksp. Teor. Fiz. 87, 663 (1984) [Sov. Phys. JETP 60, 380 (1984)].

${ }^{12}$ V.I. Mel'nikov and S.V. Meshkov, J. Chem. Phys. 85, 1018 (1986).

${ }^{13}$ L.D. Landau and E.M. Lifshitz, Mechanics, Course in Theoretical Physics, Vol. 1 (Pergamon, Oxford, 1960).

14 A. O. Caldeira and A. J. Leggett, Ann. Phys. (N.Y.) 149, 374 (1983).

${ }^{15}$ M.A. Skvortsov, Phys. Rev. B 55, 515 (1997).

${ }^{16}$ A. Krämer and M. L. Kulić, Phys. Rev. B 48, 9673 (1993).

${ }^{17}$ C. Morais Smith, B. Ivlev, and G. Blatter, Phys. Rev. B 49, 4033 (1994).

18 J. Carriga, Phys. Rev. D 49, 5497 (1994).

19 A. Ferrera, Phys. Rev. D 52, 6717 (1995).

20 J. Clarke, A. N. Cleland, M. H. Devoret, D. Esteve, and J. M. Martins, Science 239, 992 (1988).

${ }^{21}$ G.J. Dolan and J.H. Dunsmür, Physica (Amsterdam) 152B, 7 (1988).

${ }^{22}$ V.V. Moshchalkov et. al., Phys. Scr. T55, 168 (1994).

${ }^{23}$ V.V. Metlushko, M. Baert, R. Jonckheere, V.V. Moshchalkov, and Y. Bruynseraede, Solid State Commun. 91, 331 (1994).

${ }^{24}$ M. Baert, V.V. Metlushko, R. Jonckheere, V.V. Moshchalkov, and Y. Bruynseraede, Phys. Rev. Lett. 74, 3269 (1995).

${ }^{25}$ E.M. Chudnovsky, A. Ferrera, and A. Vilenkin, Phys. Rev. B 51, 1181 (1995). 